\title{
Endothelial cells-targeted soluble human Delta-like 4 suppresses both physiological and pathological ocular angiogenesis
}

\author{
YAN XianChun ${ }^{1 \dagger}$, YANG ZiYan $^{1 \dagger}, \mathrm{CHEN} \mathrm{Yan}^{2 \dagger}, \mathrm{LI} \mathrm{Na}^{1,3}$, WANG Li ${ }^{1}$, DOU GuoRui ${ }^{1,3}$, \\ LIU Yuan ${ }^{1}$, DUAN JuanLi ${ }^{1}$, FENG Lei ${ }^{1}$, DENG SanMing ${ }^{1}$, HAN Hua ${ }^{1 *}$ \& ZHANG Ping ${ }^{1 *}$ \\ ${ }^{1}$ State Key Laboratory of Cancer Biology, Department of Medical Genetics and Developmental Biology, Fourth Military Medical University, \\ $X i$ 'an 710032, China; \\ ${ }^{2}$ Department of Oncology, Xijing Hospital Fourth Military Medical University, Xi'an 710032, China; \\ ${ }^{3}$ Department of Ophthalmology, Xijing Hospital, Fourth Military Medical University, Xi'an 710032, China
}

Received October 20, 2014; accepted January 13, 2015; published online March 31, 2015

\begin{abstract}
Due to its essential roles in angiogenesis, Notch pathway has emerged as an attractive target for the treatment of pathologic angiogenesis. Although both activation and blockage of Notch signal can impede angiogenesis, activation of Notch signal may be more promising because it was shown that long-term Notch signal blockage resulted in vessel neoplasm. However, an in vivo deliverable Notch ligand with highly efficient Notch-activating capacity has not been developed. Among all the Notch ligands, Delta-like4 (Dll4) is specifically involved in angiogenesis. In this study, we generated a novel soluble Notch ligand hD4R, which consists of the Delta-Serrate-Lag-2 fragment of human Dll4 and an arginine-glycine-aspartate (RGD) motif targeting endothelial cells (ECs). We demonstrated that hD4R could bind to ECs through its RGD motif and effectively triggered Notch signaling in ECs. Further, we confirmed that hD4R could suppress angiogenesis in vitro as manifested by network formation assay and sprouting assay. More importantly, hD4R efficiently repressed neonatal retinal angiogenesis and laser-induced choroidal neovascularization $(\mathrm{CNV})$ as well in vivo. In conclusion, we have developed an in vivo deliverable Notch ligand hD4R, which suppresses angiogenesis both in vitro and in vivo, thus providing a new approach to tackle excessive angiogenesis relevant disease such as $\mathrm{CNV}$.
\end{abstract}

Notch signal, human Delta-like4, RGD, angiogenesis, choroidal neovascularization

Citation: $\quad$ Yan XC, Yang ZY, Chen Y, Li N, Dou GR, Liu Y, Duan JL, Feng L, Deng SM, Han H, Zhang P. Endothelial cells-targeted soluble human Deltalike 4 suppresses both physiological and pathological ocular angiogenesis. Sci China Life Sci, 2015, 58: 425-431, doi: 10.1007/s11427-015-4834-3

Angiogenesis is a critical process that involves the formation of new blood vessels from preexisting vessels in response to proangiogenic factors such as VEGF and FGF generated upon tissue hypoxia [1]. Angiogenesis is normally under tight control during embryogenesis and adult tissue homeostasis, and its deregulation leads to numerous disease. Among them, age-related macular degeneration (AMD) is the leading cause of irreversible blindness in those people

$\dagger$ Contributed equally to this work

*Corresponding author (email: pingzhang0622@gmail.com; huahan@fmmu.edu.cn) over 65 years of age [2]. The fast progressive "wet" form of AMD is featured by leaky excessive subretinal choroidal neovascularization (CNV), which leads to extravasations and hemorrhage into the photoreceptor cell layer, resulting in photoreceptor cell loss [2]. So far, several anti-VEGF-A antibodies have been under clinical trial to hamper the pathologic vascular outgrowth in AMD, however, the potential drug-resistance and high treatment burden call for the emergence of other novel drug candidates [2].

In metazoans, the evolutionarily conserved Notch pathway functions as an essential cell-cell contact mechanism to 
regulate numerous cell fate/lineage decisions during embryogenesis, postnatal development, and adult tissue homeostasis [3]. There are five Notch ligands, namely Jagged1, Jagged2, Delta-like 1, 3, 4 in mammals. Among them, Delta-like 4 (Dll4) is initially identified as an endotheliumspecific Notch ligand, especially in tumor vasculature [4]. Ligand binding to Notch receptors induces the proteolytic release through the gamma-secretase-catalyzed proteolysis of the Notch intracellular domain (NICD), which translocates into the nucleus and binds to the transcription factor CSL (C promoter-binding factor 1 [CBF-1]/suppressor of hairless $[\mathrm{Su}(\mathrm{H})] / \mathrm{Lin}-12$ and Glp-1 [LAG-1]). Association of NICD and CSL replaces a corepressor complex from CSL with a coactivator complex, and activates the transcription of downstream genes such as the Hes (Hairy and enhancer of split) family molecules Hey1, Hey2.

Notch signaling pathway plays vital roles in sprouting angiogenesis involving endothelial tip and stalk cell specification and also vessel maturation characterized by mural cell differentiation and recruitment $[3,5]$, thus it has been a promising target to handle pathological angiogenesis. It has been shown that both Notch activation and Notch blockade can repress angiogenesis [4]. However, compelling evidence has demonstrated that long term inhibition of Notch pathway results in vessel neoplasm and other vital toxicity [6], strengthening the idea that activating Notch signal might be an alternative choice to restrain excessive angiogenesis. Although a soluble Notch ligand is adequate for activating Notch signal in vitro, it might be insufficient to activate Notch ligand in vivo in that Notch activation relies on the endocytosis of the ligand in signal sending cells to expose the cleavage sites for $\gamma$-secretase [7]. To better induce Notch signal in vivo, we have developed a fusion protein which is composed of the DSL domain of human Dll1 (hDll1) and a nonapeptide (CRGDCGVRY) containing arginine-glycine-aspartate (RGD) [8]. RGD targets the integrin $\alpha v \beta 3$ which is expressed on endothelial cells (ECs) upon stimulation with angiogenic factors and it has been shown that the binding of RGD-containing molecular ligands with cell surface integrins triggers endocytosis $[8,9]$. We have demonstrated that hD1R could activate Notch signaling in both ECs and co-cultured human hematopoietic stem cells $[10,11]$. Because Dll4 is more specifically involved in angiogenesis [4], in this study, we extended our study to this Notch ligand. We demonstrated that hD4R can effectively trigger Notch signaling in ECs and inhibit both physiological and pathological ocular angiogenesis in vivo, thus providing a novel approach for anti-angiogenic therapy.

\section{Materials and methods}

\subsection{Expression of recombinant Trx-hD4R protein in E. coli}

The DSL domain of the hDll4 (NM_019074) cDNA frag- ment was fused with a RGD motif and amplified by PCR with a human lymphocyte cDNA library as a template. The primers are listed in Table S1 in Supporting Information. The resultant gene fragments were then cloned into pET32a(+) between the EcoR I and Sal I sites to construct pET32a-hD4R. The plasmid was transformed into the E. coli $\mathrm{BL} 21$, and the Trx-hD4R protein was induced with $0.5 \mathrm{mmol} \mathrm{L}{ }^{-1}$ isopropyl $\beta$-D-thiogalactopyranoside at $37^{\circ} \mathrm{C}$ for $12 \mathrm{~h}$. The purification of Trx-hD4R protein was accomplished by using a Ni${ }^{2+}$-NTA column (Invitrogen, USA).

\subsection{Cell culture}

Primary human umbilical vein endothelial cells (HUVECs) were maintained for 3-5 passages in an endothelial cell medium (ECM; ScienCell, USA) supplemented with 5\% FBS and endothelial cell growth supplement (ECGS). The recombinant Trx-hD4R, Trx or a $\gamma$-secretase inhibitor (GSI; Alexis Biochemicals, USA) was used at the concentration of $1 \mu \mathrm{g} \mathrm{mL}^{-1}$ or $25 \mu \mathrm{mol} \mathrm{L}{ }^{-1}$, respectively. For the cell adhesion assay, wells of 96-well dishes were coated with $5 \mu \mathrm{g}$ different recombinant proteins at $4{ }^{\circ} \mathrm{C}$ overnight, and then seeded with $1 \times 10^{5}$ HUVECs in each well. In some cases A RGD peptide (CRGDCGVRY) or a DGR peptide (CDGRCGVRY; CL Bio-Scientific, Xi'an, China) was incubated with cells for $5 \mathrm{~min}$ at the concentration of $0.5 \mathrm{mg}$ $\mathrm{mL}^{-1}$ before adding to the wells. After culturing for $10 \mathrm{~min}$, adherent cells were stained with crystal violet, photographed and subjected to image analysis.

\subsection{Western blot}

Purified recombinant protein or total cell lysates were separated by SDS-PAGE and subjected to Western blot analysis with a rabbit anti-S-tag antibody (Abcam, USA) or a rabbit anti-NICD antibody (Santa Cruz Biotechnology, USA) at $4^{\circ} \mathrm{C}$ overnight, followed by an HRP-conjugated secondary anti-rabbit IgG antibody (Millipore, USA). Membranes were developed by using the enhanced chemiluminescence system (Clinx Science Instruments, Shanghai, China).

\subsection{Immunofluorescence}

Cultured cells were fixed by $4 \%$ paraformaldehyde (PFA) for $30 \mathrm{~min}$ at room temperature and blocked with $1 \%$ bovine serum albumin (BSA) and $0.5 \%$ Triton $\mathrm{X}-100$ in phosphate-buffered saline (PBS) for $2 \mathrm{~h}$, then incubated at $4^{\circ} \mathrm{C}$ overnight with the rabbit anti-S-tag or rabbit anti-NICD. After washing, cells were incubated with a Cy3-conjugated goat anti-rabbit IgG secondary antibody (Sigma, USA). For tissue staining, retina or choroid samples were fixed overnight at $4{ }^{\circ} \mathrm{C}$ with $4 \%$ PFA, blocked and permeabilized, then immunostained with FITC-labeled GSL I-isolectin B4 (Vector Laboratories, USA) at $4^{\circ} \mathrm{C}$ overnight. Images were captured with a fluorescence or confocal microscope. 


\subsection{Quantitative reverse transcription (RT)-PCR}

Total RNA was isolated with a TRIzol reagent (Invitrogen, USA) according to the manufacturer's instructions. cDNA was synthesized by a reverse transcription system (TaKaRa, Dalian, China). MRNA expression levels were quantified using a kit (SYBR Premix EX Taq, TaKaRa), detected by ABPRI ISM 7500 Real-Time PCR System. The PCR primers are listed in Table S1 in Supporting Information.

\subsection{Endothelial network formation assay}

The 48-well dishes were pre-coated by adding $200 \mu \mathrm{L}$ Matrigel Basement Membrane Matrix (BD Biosciences, USA) and incubated at $37^{\circ} \mathrm{C}$ for $40 \mathrm{~min}$. HUVECs were then seeded on the gel together with $1.25 \mu \mathrm{g} \mathrm{mL}{ }^{-1}$ Trx or Trx-hD4R, and incubated at $37^{\circ} \mathrm{C}$ for $6 \mathrm{~h}$. Images were observed and the branches and the length of the cell cords of the enclosed lumens were measured.

\subsection{Fibrin bead assay}

The microbeads carrying 400 cells per bead were embedded in the fibrinogen containing thrombin $\left(0.625 \mathrm{U} \mathrm{mL}^{-1}\right)$, and then $0.5 \mathrm{~mL}$ of EGM-2 medium was added with lung fibroblasts as described earlier [10]. Trx or Trx-hD4R was added at the concentration of $0.5 \mu \mathrm{g} /$ well every other day. Sprouting was quantified by measuring the number and length of sprouts 4 days later.

\subsection{In vivo application of recombinant proteins}

Postnatal day 3 (P3) pups were intravitreally injected with Trx or Trx-hD4R $(0.1 \mu \mathrm{g})$ and then subcutaneously injected with Trx or Trx-hD4R ( $5 \mu \mathrm{g})$ once a day for $4 \mathrm{~d}$. Eyes were collected on P7 and fixed in $4 \%$ PFA at $4{ }^{\circ} \mathrm{C}$ overnight, and stained as described previously [12]. All animal experiments were approved by the Animal Experiment Administration Committee of the University.

\subsection{Laser-induced CNV assay}

Laser-induced CNV assay was performed as described [12]. C57BL/6J mice were anesthetized and the pupils were dilated. Six laser burns were performed at the 2-, 4-, 6-, 8-, $10-$, and 12-o'clock positions of the posterior pole around the optic nerve of both eyes. Mice were then injected through the tail vein with of $25 \mu \mathrm{g}$ of Trx-hD4R or Trx on days 1, 3, and 5 after laser irradiation. Eyes were collected and stained on day 7 .

\subsection{Statistics}

Statistical analysis was performed with the Image Pro Plus 6.0 and Graph Pad Prism 5 software. Student's $t$ test was used for statistical analyses (unless otherwise specified). Data were expressed as mean \pm standard deviation (SD). $P<0.05$ was considered statistically significant.

\section{Results}

\subsection{Cloning, expression and purification of the recom- binant protein $\mathrm{hD} 4 \mathrm{R}$}

The recombinant hD4R contained amino acid 121-221 of human Notch ligand Dll4 (including the DSL domain responsible for Notch receptor binding) and a RGD motif targeting ECs (Figure S1A in Supporting Information). The 333 bp cDNA fragment encoding the DSL domain of hDll4 fused with the RGD-encoding sequence was amplified from a human lymphocyte cDNA library by PCR (Figure S1B in Supporting Information). The resulting fragment was subcloned into prokaryotic expression vector pET32a(+) containing the Trx-His-S tag, and recombinants were identified with enzyme digestion using $\mathrm{Sal}$ I and $\mathrm{Bgl}$ II, which generated fragments of 5809 and 424 bp as expected (Figure S1C in Supporting Information). To produce the recombinant proteins, E. coli BL21 was transformed with pET32a-hD4R or the control vector. Then the expression of the recombinant proteins was induced with $0.5 \mathrm{mmol} \mathrm{L}^{-1}$ isopropyl $\beta$-D-thiogalactoside (IPTG) for $12 \mathrm{~h}$ at $37^{\circ} \mathrm{C}$. SDS-PAGE analysis of protein extracts of total bacteria, the supernatants or the precipitates revealed that the recombinant protein hD4R mainly existed in the precipitation fraction (Figure S1D in Supporting Information). The recombinant protein was then purified with $\mathrm{Ni}^{2+}$-NTA affinity chromatography (Figure S1E in Supporting Information). We detected the presence of a specific band of about $33 \mathrm{kD}$ by Western blot with the anti-S-tag antibody, consistent with the estimated molecular weight (32.3 kD) of hD4R (Figure S1F in Supporting Information).

\section{2 hD4R could bind to ECs specifically}

To verify whether hD4R could bind to ECs, the capability of HUVECs to adhere to culture dishes pre-coated with hD4R or Trx was evaluated in a cell adhesion assay. Much more HUVECs were found to adhere to culture dishes pre-coated with hD4R than to dishes pre-coated with Trx (Figure 1A and B). Moreover, the adherence of HUVECs to the hD4R-coated dishes could be blocked by a synthetic RGD nonapeptide but not by a DGR nonapeptide (Figure $1 \mathrm{C}$ and $\mathrm{D})$. Then the capability of hD4R binding to ECs was further examined with an in vitro binding experiment followed by detection with immunofluorescence analysis. HUVECs and lung fibroblasts were incubated with hD4R and then subjected to indirect immunofluorescent staining with an anti-S-tag antibody. The result showed that hD4R could bind to HUVECs more strongly than to lung fibroblasts (Figure $1 \mathrm{E}$ and $\mathrm{F}$ ). These results verified that hD4R 
A

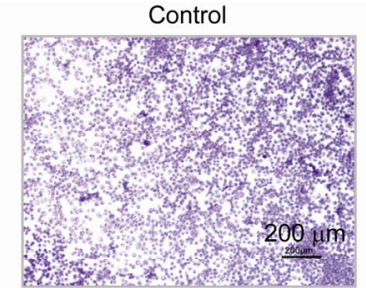

C

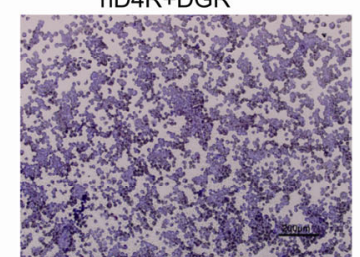

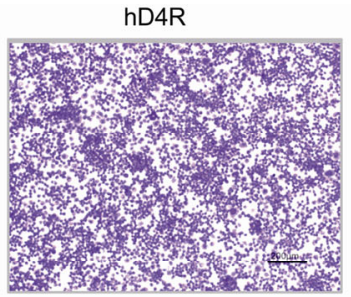

$\mathrm{hD} 4 \mathrm{R}+\mathrm{RGD}$

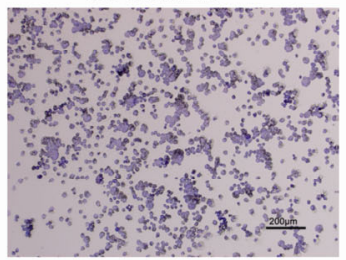

S-Tag
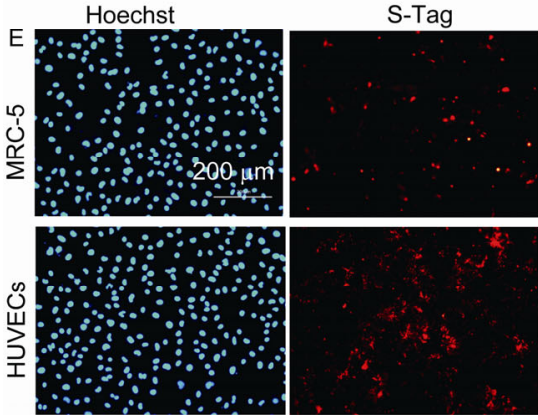

Merged

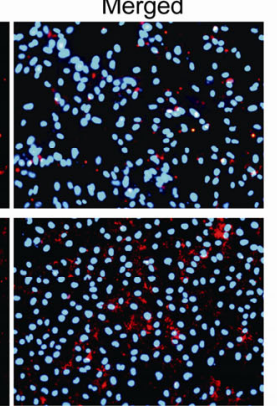

B

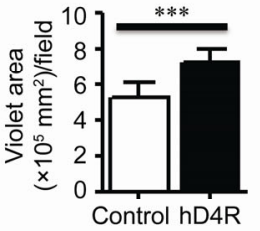

D
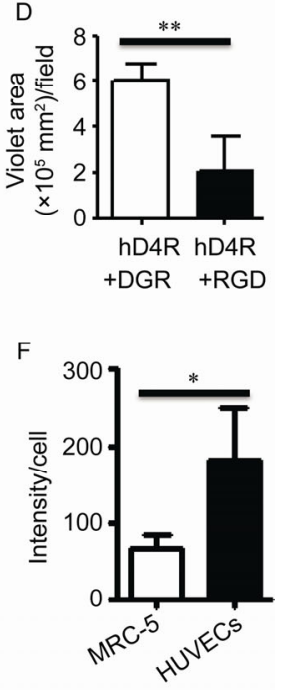

Figure 1 hD4R could efficiently bind to ECs through the RGD motif. A, HUVECs were plated in wells pre-coated with Trx or hD4R. After incubation, adherent cells were stained with crystal violet and photographed. B, Area of stained crystal violet of each field was quantified and compared ( $n=6$ ). C, HUVECs were incubated with synthetic DGR or RGD nonapeptide and then distributed into wells pre-coated with hD4R. After incubation, adherent cells were stained with crystal violet and photographed. D, Area of stained cells per field was measured and compared ( $n=6)$. E, HUVECs and lung fibroblasts were incubate with hD4R and then detected by the anti-S-tag antibody. F, The intensity of fluorescent signals per cell was quantified and compared ( $n=5)$. Bars represent mean $\pm \mathrm{SD} ; *, P<0.05 ; * *, P<0.01 ; * * *, P<0.001$.

could efficiently bind to ECs, most likely through the RGD motif.

\section{3 hD4R activated Notch signaling in ECs}

To evaluate whether hD4R could activate Notch signaling in ECs, hD4R- or Trx-treated HUVECs were subjected to immunofluorescence staining for the cleaved Notch1 intercellular domain which is an indication of the activation of Notch signaling. Clearly, there were much more positive staining signals in the hD4R-treated HUVECs than in the control cells, which were largely abolished by the administration of GSI (Figure 2A and B). Consistently, in HUVECs incubated with hD4R, Western blot analysis displayed a significant increase in the level of NICD, which was mostly abrogated by GSI (Figure 2C). Furthermore, the mRNA level of Hey1, a major Notch downstream target in ECs, increased remarkably in hD4R-treated HUVECs (Figure 2D). In addition, hD4R-treated HUVECs displayed a significant increase in the expression of VEGFR1 and decrease in the expression of VEGFR3 (Figure 2D), in line with previous reports $[13,14]$. These results demonstrated that hD4R could effectively trigger Notch signaling in ECs, thus being a functional Notch ligand in vitro.

\section{4 hD4R inhibited angiogenesis in vitro}

We then employed in vitro assays to examine the effects of $\mathrm{hD} 4 \mathrm{R}$ on lumen formation and angiogenic sprouting by ECs. Firstly, HUVECs were cultured in a Matrigel Basement Membrane Matrix in the presence of Trx or hD4R, and the formation of endothelial networks was compared by counting network branches and branch length per field. Compared with the control, hD4R-treated HUVECs displayed a smaller amount of branches and much shorter branch length in each field, indicating that hD4R remarkably inhibited HUVECs network formation (Figure $3 \mathrm{~A}$ and $\mathrm{B}$ ). Then, in a fibrin gel bead assay which mimicked the angiogenesis process in vivo, the sprouting capability of ECs could be evaluated by the numbers of sprouts on each bead and the length of each sprout. As shown in Figure $3 \mathrm{C}$ and D, there was a remarkable reduction in the number of sprouts per bead and a dramatic decrease in the sprout length in hD4R-treated HUVECs, compared with that of control HUVECs. The result indicated that hD4R could effectively suppress the sprouting and elongation of sprouting buds of 

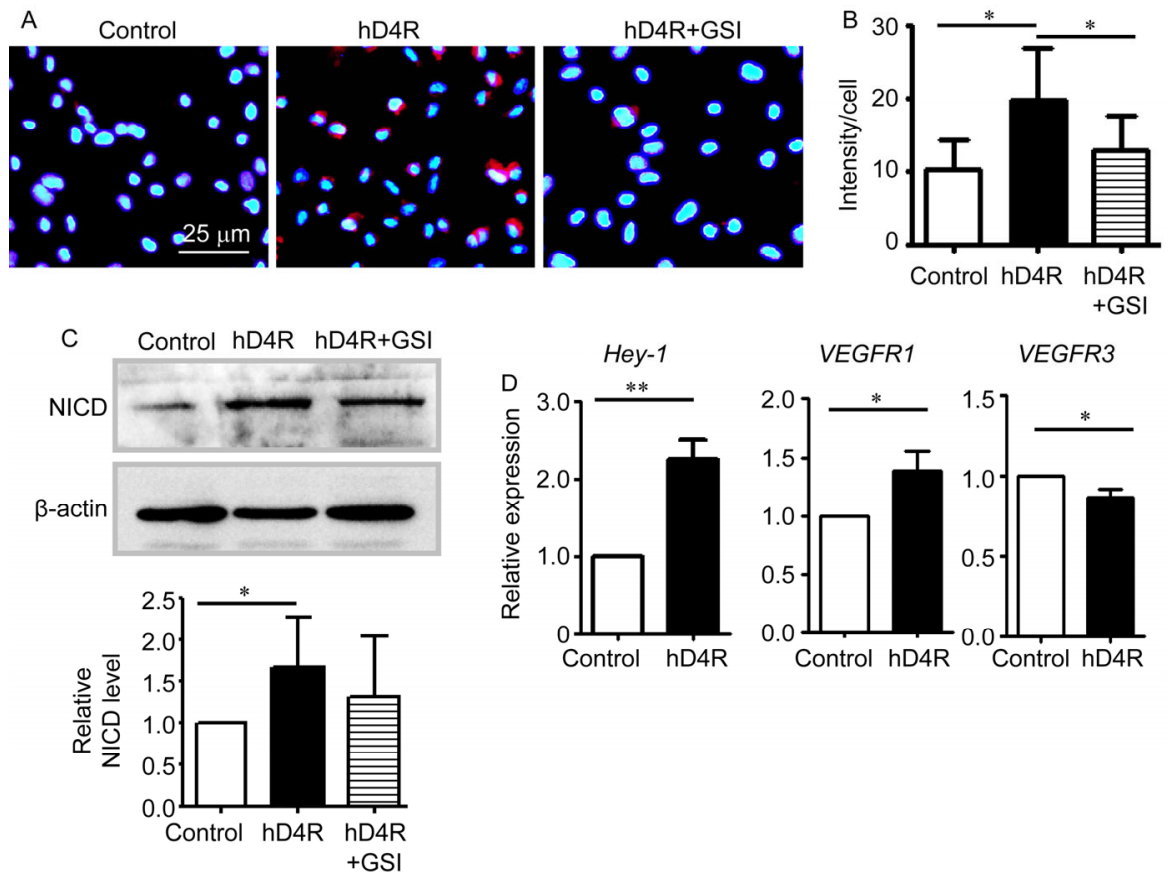

Figure 2 hD4R could efficiently trigger Notch signaling. A, hD4R activated Notch signaling in a GSI-dependent manner. HUVECs were incubated with Trx, Trx-hD4R or Trx-hD4R plus GSI. NICD level was determined by immunofluorescence staining using the anti-NICD antibody. B, The intensity of fluorescent signals per cell was measured and compared $(n=6)$. C, Western blot. HUVECs were treated as in A. Western blot analysis of NICD was performed by using the anti-NICD antibody, with $\beta$-actin as an internal control. The bands were quantified and compared $(n=6)$. D, HUVECs were treated as in A. Then the cells were harvested and the expression of Hey1, VEGFR1, and VEGFR3 was detected by quantitative RT-PCR $(n=9)$. Bars represent mean \pm SD; *, $P<0.05$; **, $P<0.01$.
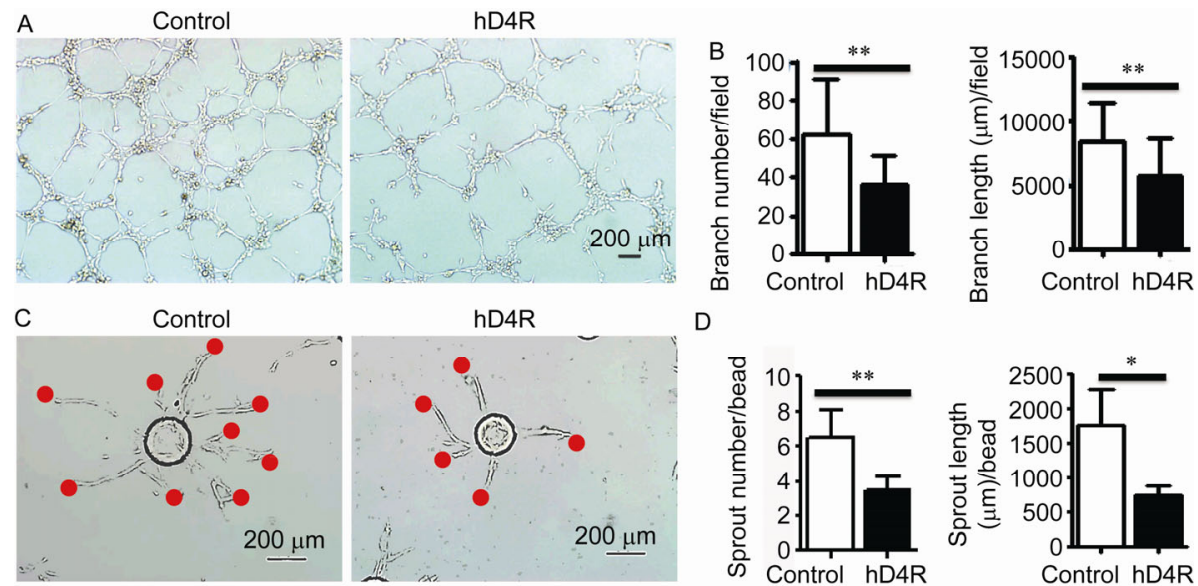

D
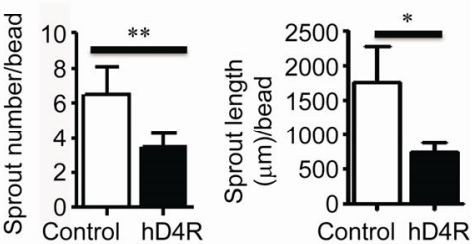

Figure 3 hD4R could suppress angiogenesis in vitro. A, HUVECs were cultured on the Matrigel Basement Membrane Matrix in the presence of Trx or hD4R. Cells were cultured for $6 \mathrm{~h}$ for the formation of cell networks, and photographed. B, The formation of the endothelial networks was quantified by counting the number and length of branches per field and compared ( $n=6)$. C, HUVECs were mixed with microbeads and cultured for $96 \mathrm{~h}$ for angiogenic sprouting in the presence of Trx or hD4R. D, The numbers of sprouts and the sprout length per bead were counted and compared ( $n=5)$. Bars represent mean $\pm \mathrm{SD} ; *, P<0.05 ; * *, P<0.01$.

ECs. Collectively, these data indicated that hD4R repressed angiogenesis in vitro.

\section{5 hD4R suppressed angiogenesis in vivo}

We next examined the effects of $\mathrm{hD} 4 \mathrm{R}$ on neonatal retinal angiogenesis. The retinal surface angiogenesis begins on early postnatal (P1) stage in mice and spreads from the optic stalk to the retinal periphery via gradient vascular development [12]. So pups were first intravitreally injected and then subcutaneously injected with Trx or hD4R once a day for $4 \mathrm{~d}$, and the angiogenic sprouting of retinal vasculature was examined on P7 by whole-mount retinal staining with isolectin B4. The distal, middle, and proximal regions of the 
retinal vascular bed were examined separately for changes in vascular density and branch points. Compared with the control, hD4R-treated mice displayed a less condensed vessel network and a decrease in vascular loops in the distal, middle and proximal areas (Figure 4A and B). Furthermore, the fronts of sprouting have less tip cells (Figure 4C). These in vivo data demonstrated that hD4R suppressed physiological angiogenesis.

Laser coagulation of the mouse choroid in C57BL/6J mice induced rupture of Bruchs membrane and subsequent $\mathrm{CNV}$, representing a well recognized animal model for "wet" AM [5]. To test whether local administration of hD4R could hinder the progression of $\mathrm{CNV}$, adult C57BL/6J were injected with Trx or hD4R after laser photocoagulation on days 1,3 , and 5 , then $\mathrm{CNV}$ was detected by staining sections of choroid-sclera complexes through the laser spots with isolectin B4. Compared with the controls, a significant decrease in $\mathrm{CNV}$ area in mice treated with hD4R was documented (Figure 4D), which demonstrated that $\mathrm{hD} 4 \mathrm{R}$ could inhibit pathological ocular angiogenesis such as CNV. Collectively, these data indicated that hD4R suppressed both physiological and pathological ocular angiogenesis.

\section{Discussion}

The role of Notch in sprouting angiogenesis has been well defined by accumulating evidence. In the absence of Notch signaling ECs continue to form sprouts in response to VEGF, resulting in extra sprouts, as has been documented in different model systems such as zebrafish embryos, mouse retina and xenograft tumors [3,15]. Enforced Notch signaling represses angiogenesis both in vitro and in vivo $[4,5,15]$. Thus, Notch pathway has been a promising target to interfere with to benefit angiogenesis-related disease. Results from a number of studies using either a Dll4specific neutralizing antibody [16], a Notch1-specific antagonistic antibody [17], a soluble Dll4 fusion protein [18], or a soluble Notch fusion protein have confirmed profound impact of Notch signaling on angiogenesis, especially in tumors [5]. These studies have tried to block Notch signals for intervening with excessive angiogenesis, but meets great challenge of long-term toxicity. Skin lesions with features of vascular neoplasms were observed in male rats after 8 weeks of continuous anti-Dll4 exposure [6]. Goblet cell metaplasia and complete blockade of $\mathrm{T}$ cell development coupled with ectopic appearance of immature B cells in the thymus were the major on-target toxicities of inhibiting Notch signaling [19]. On the other hand, enforced Notch signal also represses angiogenesis by restraining the number of tip cells and hindering EC proliferation [15]. Moreover, enhanced Notch signal promotes the coverage of mural cells and enhances EC adhesion, contributing to the improvement of vessel perfusion and function [20,21]. Thus, activation of Notch signal seems to be more promising than blockage of Notch signal in preventing excessive angiogenesis, especially for controlling neovascular AMD.
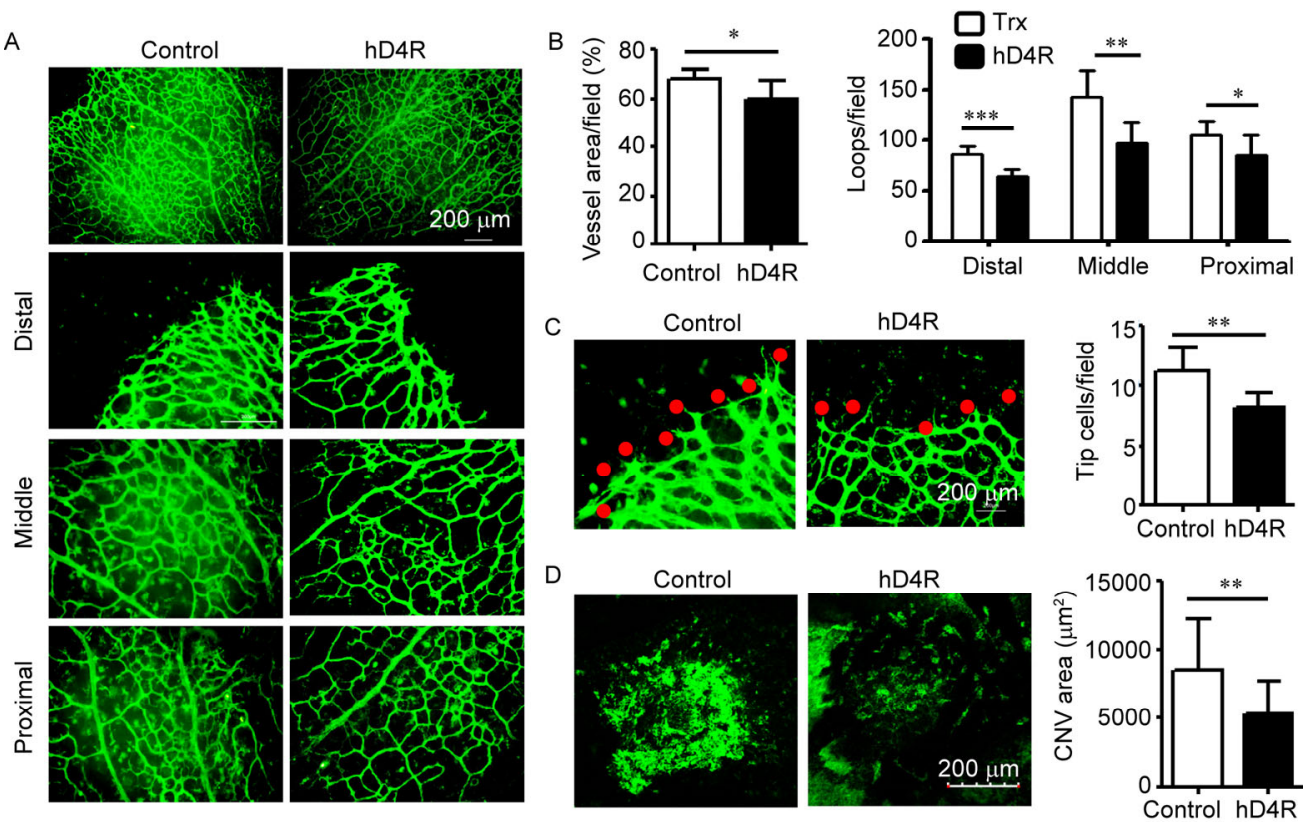

Figure 4 hD4R could hamper angiogenesis in vivo. A and B, P3 pups were treated with Trx or hD4R. Then on P7, the retinas were stained with FITC-labeled GSL I-isolectin B4. The structures of the whole, distal, middle and proximal retinal vasculature were shown in (A). The numbers of enclosed capillary loops and vessel area were compared (B) $(n=6)$. C and D, Tip cells at the angiogenic fronts were counted and compared ( $n=6$ ). E and F, Representative confocal images showing repression of CNV by hD4R compared with the Trx control. CNV area ( $\left.\mu \mathrm{m}^{2}\right)$ was quantified by using the Image pro plus 6.0 ( $n=16)$. Bars represent mean $\pm \mathrm{SD} ; *, P<0.05$; **, $P<0.01$. 
The activation of Notch signaling is dependent on binding with ligands, a protein family composed of three Delta and two Jagged members in mammals [4]. Among them, Dll4 is endothelium-specific and mainly responsible for initiating and maintaining a stalk cell fate to restrict sprouting angiogenesis by counteracting VEGF signal. The extracellular domain of Dll4 is composed of a N-terminal signal peptide domain, a DSL domain and multiple EGF repeats, in which DSL domain is responsible for binding to Notch receptor and has been reported to be adequate for activating Notch signal [4]. Although the DSL domain of Notch ligand is sufficient to activate Notch signal in vitro, it might not be highly efficient in vivo in that efficient Notch activation depends on endocytosis of the ligand in signal sending cells.

RGD is a well-known peptide binding to endothelial specific $\alpha v \beta 3$ integrin and RGD targeted molecules could stimulate endocytosis, which is required for Notch activation [8]. Based on these reasons, we choose to express a fusion protein containing the DSL domain of hDll4 and a RGD peptide, which is soluble and can stimulate endocytosis after binding to ECs through the integrin. In this study, we documented that hD4R specifically binds to Notch receptors and efficiently activate Notch signaling as manifested by increased intracellular NICD level and up-regulated downstream gene expression. Furthermore, we demonstrated that this fusion protein could dramatically suppress the network formation capability and sprouting angiogenesis of HUVECs, indicating that it is functional in vitro. More importantly and encouragingly, although it could only mildly repress neonatal retinal angiogenesis, it greatly hindered the laser-induced $\mathrm{CNV}$ in mice, making it a promising treatment of vessel outgrowth-related decease such as AMD. All these data have demonstrated that this novel fusion protein could work as an efficient Notch ligand in vivo. In summary, we have developed a novel functional Notch ligand hD4R that is endothelial targeted and highly efficient in vivo, thus providing a novel approach for anti-angiogenesis therapy. However, critical pharmaceutical characteristics of this fusion protein, including its half-life in vivo and short-term or long-term toxicity, need to be carefully investigated before it could be tested clinically.

The authors declare that they have no potential conflict of interest.

This work was supported by the National Natural Science Foundation of China (31370769, 81200707, 91339115, 31301194).

1 Potente M, Gerhardt H, Carmeliet P. Basic and therapeutic aspects of angiogenesis. Cell, 2011, 146: 873-887

2 Lally DR, Gerstenblith AT, Regillo CD. Preferred therapies for neovascular age-related macular degeneration. Curr Opin Ophthalmol, 2012, 23: 182-188

3 Benedito R, Hellstrom M. Notch as a hub for signaling in angiogenesis. Exp Cell Res, 2013, 319: 1281-1288

4 Kume T. Ligand-dependent Notch signaling in vascular formation. Adv Exp Med Biol, 2012, 727: 210-222

5 Dou GR, Wang L, Wang YS, Han H. Notch signaling in ocular vasculature development and diseases. Mol Med, 2012, 18: 47-55

6 Yan M, Callahan CA, Beyer JC, Allamneni KP, Zhang G, Ridgway JB, Niessen K, Plowman GD. Chronic DLL4 blockade induces vascular neoplasms. Nature, 2010, 463: E6-7

7 Fortini ME, Bilder D. Endocytic regulation of Notch signaling. Curr Opin Genet Dev, 2009, 19: 323-328

8 Danhier F, Le Breton A, Preat V. RGD-based strategies to target alpha(v) beta(3) integrin in cancer therapy and diagnosis. Mol Pharm, 2012, 9: 2961-2973

9 Caswell PT, Vadrevu S, Norman JC. Integrins: masters and slaves of endocytic transport. Nat Rev Mol Cell Biol, 2009, 10: 843-853

10 Zhao XC, Dou GR, Wang L, Liang L, Tian DM, Cao XL, Qin HY, Wang CM, Zhang P, Han $\mathrm{H}$. Inhibition of tumor angiogenesis and tumor growth by the DSL domain of human Delta-like 1 targeted to vascular endothelial cells. Neoplasia, 2013, 15: 815-825

11 Tian DM, Liang L, Zhao XC, Zheng MH, Cao XL, Qin HY, Wang CM, Liang YM, Han H. Endothelium-targeted Delta-like 1 promotes hematopoietic stem cell expansion ex vivo and engraftment in hematopoietic tissues in vivo. Stem Cell Res, 2013, 11: 693-706

12 Dou GR, Wang YC, Hu XB, Hou LH, Wang CM, Xu JF, Wang YS, Liang YM, Yao LB, Yang AG, Han H. RBP-J, the transcription factor downstream of Notch receptors, is essential for the maintenance of vascular homeostasis in adult mice. FASEB J, 2008, 22: 1606-1617

13 Benedito R, Rocha SF, Woeste M, Zamykal M, Radtke F, Casanovas O, Duarte A, Pytowski B, Adams RH. Notch-dependent VEGFR3 upregulation allows angiogenesis without VEGF-VEGFR2 signalling. Nature, 2012, 484: 110-114

14 Hellstrom M, Phng LK, Hofmann JJ, Wallgard E, Coultas L, Lindblom P, Alva J, Nilsson AK, Karlsson L, Gaiano N, Yoon K, Rossant J, Iruela-Arispe ML, Kalen M, Gerhardt H, Betsholtz C. Dll4 signalling through Notch1 regulates formation of tip cells during angiogenesis. Nature, 2007, 445: 776-780

15 Zhang P, Yan X, Chen Y, Yang Z, Han H. Notch signaling in blood vessels: from morphogenesis to homeostasis. Sci China Life Sci, 2014, 57: 774-780

16 Ridgway J, Zhang G, Wu Y, Stawicki S, Liang WC, Chanthery Y, Kowalski J, Watts RJ, Callahan C, Kasman I, Singh M, Chien M, Tan C, Hongo JA, de Sauvage F, Plowman G, Yan M. Inhibition of D114 signalling inhibits tumour growth by deregulating angiogenesis. Nature, 2006, 444: 1083-1087

17 Wu Y, Cain-Hom C, Choy L, Hagenbeek TJ, de Leon GP, Chen Y, Finkle D, Venook R, Wu X, Ridgway J, Schahin-Reed D, Dow GJ, Shelton A, Stawicki S, Watts RJ, Zhang J, Choy R, Howard P, Kadyk L, Yan M, Zha J, Callahan CA, Hymowitz SG, Siebel CW. Therapeutic antibody targeting of individual Notch receptors. Nature, 2010, 464: 1052-1057

18 Noguera-Troise I, Daly C, Papadopoulos NJ, Coetzee S, Boland P, Gale NW, Lin HC, Yancopoulos GD, Thurston G. Blockade of D114 inhibits tumour growth by promoting non-productive angiogenesis. Nature, 2006, 444: 1032-1037

19 Yan M. Therapeutic promise and challenges of targeting DLL4/NOTCH1. Vasc Cell, 2011, 3: 17

20 Scheppke L, Murphy EA, Zarpellon A, Hofmann JJ, Merkulova A, Shields DJ, Weis SM, Byzova TV, Ruggeri ZM, Iruela-Arispe ML, Cheresh DA. Notch promotes vascular maturation by inducing integrin-mediated smooth muscle cell adhesion to the endothelial basement membrane. Blood, 2012, 119: 2149-2158

21 Bentley K, Franco CA, Philippides A, Blanco R, Dierkes M, Gebala V, Stanchi F, Jones M, Aspalter IM, Cagna G, Westrom S, Claesson-Welsh L, Vestweber D, Gerhardt H. The role of differential VE-cadherin dynamics in cell rearrangement during angiogenesis. Nat Cell Biol, 2014, 16: 309-321

Open Access This article is distributed under the terms of the Creative Commons Attribution License which permits any use, distribution, and reproduction in any medium, provided the original author(s) and source are credited. 